\title{
Pure Links Between Graph Invariants and Large Cycle Structures
}

\author{
Zh.G. Nikoghosyan* \\ Institute for Informatics and Automation Problems, \\ National Academy of Sciences, \\ Armenia
}

\section{Introduction}

Hamiltonian graph theory is one of the oldest and attractive fields in discrete mathematics, concerning various path and cycle existence problems in graphs. These problems mainly are known to be NP-complete that force the graph theorists to direct efforts toward understanding the global and general relationship between various invariants of a graph and its path and cycle structure.

This chapter is devoted to large cycle substructures, perhaps the most important cycle structures in graphs: Hamilton, longest and dominating cycles and some generalized cycles including Hamilton and dominating cycles as special cases.

Graph invariants provide a powerful and maybe the single analytical tool for investigation of abstract structures of graphs. They, combined in convenient algebraic relations, carry global and general information about a graph and its particular substructures such as cycle structures, factors, matchings, colorings, coverings, and so on. The discovery of these relations is the primary problem of graph theory.

In the literature, eight basic (initial) invariants of a graph $G$ are known having significant impact on large cycle structures, namely order $n$, size $q$, minimum degree $\delta$, connectivity $\kappa$, independence number $\alpha$, toughness $\tau$ and the lengths of a longest path and a longest cycle in $G \backslash C$ for a given longest cycle $C$ in $G$, denoted by $\bar{p}$ and $\bar{c}$, respectively.

In this chapter we have collected 37 pure algebraic relations between $n, q, \delta, \kappa, \alpha, \tau, \bar{p}$ and $\bar{c}$ ensuring the existence of a certain type of large cycles. The majority of these results are sharp in all respects.

Focusing only on basic graph invariants, as well as on pure algebraic relations between these parameters, in fact, we present the simplest kind of relations for large cycles having no forerunners in the area. Actually they form a source from which nearly all possible hamiltonian results (including well-known Ore's theorem, Pósa's theorem and many other

${ }^{*}$ G.G. Nicoghossian (up to 1997) 
generalizations) can be developed further by various additional new ideas, generalizations, extensions, restrictions and structural limitations:

- generalized and extended graph invariants - degree sequences (Pósa type, Chvátal type), degree sums (Ore type, Fun type), neighborhood unions, generalized degrees, local connectivity, and so on,

- extended list of path and cycle structures - Hamilton, longest and dominating cycles, generalized cycles including Hamilton and dominating cycles as special cases, 2-factor, multiple Hamilton cycles, edge disjoint Hamilton cycles, powers of Hamilton cycles, $k$ ordered Hamilton cycles, arbitrary cycles, cycle systems, pancyclic-type cycle systems, cycles containing specified sets of vertices or edges, shortest cycles, analogous path structures, and so on,

- $\quad$ structural (descriptive) limitations - regular, planar, bipartite, chordal and interval graphs, graphs with forbidden subgraphs, Boolean graphs, hypercubes, and so on,

- graph extensions - hypergraphs, digraphs and orgraphs, labeled and weighted graphs, infinite graphs, random graphs, and so on.

We refer to (Bermond, 1978) and (Gould, 1991, 2003) for more background and general surveys.

The order $n$, size $q$ and minimum degree $\delta$ clearly are easy computable graph invariants. In (Even \& Tarjan, 1975), it was proved that connectivity $\kappa$ can be determined in polynomial time, as well. Determining the independence number $\alpha$ and toughness $\tau$ are shown in (Garey \& Johnson, 1983) and (Bauer et al., 1990a) to be $C D_{\lambda}$-hard problems. Moreover, it was proved (Bauer et al., 1990a) that for any positive rational number $t$, recognizing $t$-tough graphs (in particular 1-tough graphs) is an NP -hard problem.

The order $n$ and size $q$ are neutral with respect to cycle structures. Meanwhile, they become more effective combined together (Theorem 1). The minimum degree $\delta$ having high frequency of occurrence in different relations is, in a sense, a more essential invariant than the order and size, providing some dispersion of the edges in a graph. The combinations between order $n$ and minimum degree $\delta$ become much more fruitful especially under some additional connectivity conditions. The impact of some relations on cycle structures can be strengthened under additional conditions of the type $\delta \geq \alpha \pm i$ for appropriate integer $i$. By many graph theorists, the connectivity $\kappa$ is at the heart of all path and cycle questions providing comparatively more uniform dispersion of the edges. An alternate connectedness measure is toughness $\tau$ - the most powerful and less investigated graph invariant introduced by Chvátal (Chvátal, 1973) as a means of studying the cycle structure of graphs. Chvátal (Chvátal, 1973) conjectured that there exists a finite constant $t_{0}$ such that every $t_{0}$-tough graph is hamiltonian. This conjecture is still open. We have omitted a number of results involving toughness $\tau$ as a parameter since they are far from being best possible.

Large cycle structures are centered around well-known Hamilton (spanning) cycles. Other types of large cycles were introduced for different situations when the graph contains no Hamilton cycles or it is difficult to find it. Generally, a cycle $C$ in a graph $G$ is a large cycle if it dominates some certain subgraph structures in $G$ in a sense that every such structure 
has a vertex in common with $C$. When $C$ dominates all vertices in $G$ then $C$ is a Hamilton cycle. When $C$ dominates all edges in $G$ then $C$ is called a dominating cycle introduced by Nash-Williams (Nash-Williams, 1971). Further, if $C$ dominates all paths in $G$ of length at least some fixed integer $\lambda$ then $C$ is a $P D_{\lambda}$ (path dominating)-cycle introduced by Bondy (Bondy, 1981). Finally, if $C$ dominates all cycles in $G$ of length at least $\lambda$ then $C$ is a $C D_{\lambda}$ (cycle dominating)-cycle, introduced in (Zh.G. Nikoghosyan, 2009a). The existence problems of generalized $P D_{\lambda}$ and $C D_{\lambda}$-cycles are studied in (Zh.G. Nikoghosyan, 2009a) including Hamilton and dominating cycles as special cases.

Section 2 is devoted to necessary notation and terminology. In Section 3, we discuss pure relations between various basic invariants of a graph and Hamilton cycles. Next sections are devoted to analogous pure relations concerning dominating cycles (Section 4 ), $C D_{\lambda}$-cycles (Section 5), long cycles (Section 6), long cycles with Hamilton cycles (Section 7), long cycles with dominating cycles (Section 8) and long cycles with $C D_{\lambda}$-cycles (Section 9). In Section 10 we present the proofs of Theorems 6, 21 and 27. Concluding remarks are given in Section 11.

\section{Terminology}

We consider only finite undirected graphs without loops or multiple edges. A good reference for any undefined terms is (Bondy \& Murty, 1976). The set of vertices of a graph $G$ is denoted by $V(G)$ and the set of edges by $E(G)$. For $S$ a subset of $V(G)$, we denote by $G \backslash S$ the maximum subgraph of $G$ with vertex set $V(G) \backslash S$. For a subgraph $H$ of $G$ we use $G \backslash H$ short for $G \backslash V(H)$. Denote by $N(x)$ the neighborhood of a vertex $x$ in $G$. Put $d(x)=|N(x)|$

A simple cycle (or just a cycle) $C$ of length $t$ is a sequence $v_{1} v_{2} \ldots v_{t} v_{1}$ of distinct vertices $v_{1}, v_{2}, \ldots, v_{t}$ with $v_{i} v_{i+1} \in E(G)$ for each $i \in\{1, \ldots, t\}$, where $v_{t+1}=v_{1}$. When $t=2$, the cycle $C=v_{1} v_{2} v_{1}$ on two vertices $v_{1}, v_{2}$ coincides with the edge $v_{1} v_{2}$, and when $t=1$, the cycle $C=v_{1}$ coincides with the vertex $v_{1}$. So, all vertices and edges in a graph can be considered as cycles of lengths 1 and 2, respectively. A graph $G$ is hamiltonian if $G$ contains a Hamilton cycle, i.e. a cycle containing all vertices of $G$. Let $\lambda$ be an integer. A cycle $C^{\prime}$ in $G$ is a $P D_{\lambda}$-cycle if $|P| \leq \lambda-1$ for each path $P$ in $G \backslash C^{\prime}$ and is a $C D_{\lambda}$-cycle if $\left|C^{\prime \prime}\right| \leq \lambda-1$ for each cycle $C^{\prime \prime}$ in $G \backslash C^{\prime}$. In particular, $P D_{0}$-cycles and $C D_{1}$-cycles are well-known Hamilton cycles and $P D_{1}$-cycles and $C D_{2}$-cycles are often called dominating cycles.

We reserve $n, q, \delta, \kappa$ and $\alpha$ to denote the number of vertices (order), number of edges (size), minimum degree, connectivity and independence number of a graph, respectively. Let $c$ denote the circumference - the length of a longest cycle in a graph. In general, $c \geq 1$. For $C$ a longest cycle in $G$, denote by $\bar{p}$ and $\bar{c}$ the lengths of a longest path and a longest cycle in $G \backslash C$, respectively. Let $s(G)$ denote the number of components of a graph $G$. A graph $G$ is $t$-tough if $|S| \geq t \cdot s(G \backslash S)$ for every subset $S \subseteq V(G)$ with $s(G \backslash S)>1$. The toughness of $G$, denoted $\tau(G)$, is the maximum value of $t$ for which $G$ is $t$-tough (taking $\tau\left(K_{n}\right)=\infty$ for all $n \geq 1)$. 
An $(x, y)$-path is a path with end vertices $x$ and $y$. Given an $(x, y)$-path $L$ of $G$, we denote by $\vec{L}$ the path $L$ with an orientation from $x$ to $y$. If $u, v \in V(L)$ then $u \vec{L} v$ denotes the consecutive vertices on $\vec{L}$ from $u$ to $v$ in the direction specified by $\vec{L}$. The same vertices, in reverse order, are given by $v \overleftarrow{L} u$. For $\vec{L}=x \vec{L} y$ and $u \in V(L)$, let $u^{+}(\vec{L})$ (or just $\left.u^{+}\right)$denotes the successor of $u(u \neq y)$ on $\vec{L}$, and $u^{-}$denotes its predecessor $(u \neq x)$. If $A \subseteq V(L) \backslash\{y\}$ then we denote $A^{+}=\left\{v^{+} \mid v \in A\right\}$. Similar notation is used for cycles. If $Q$ is a cycle and $u \in V(Q)$, then $u \vec{Q} u=u$.

Let $a, b, t, k$ be integers with $k \leq t$. We use $H(a, b, t, k)$ to denote the graph obtained from $t K_{a}+\bar{K}_{t}$ by taking any $k$ vertices in subgraph $\bar{K}_{t}$ and joining each of them to all vertices of $K_{b}$. Denote by $L_{\delta}$ the graph obtained from $3 K_{\delta}+K_{1}$ by taking one vertex in each of three copies of $K_{b}$ and joining them each to other. For odd $n$, where $n \geq 15$, construct the graph $G_{n}$ from $\bar{K}_{(n-1) / 2}+K_{\delta}+K_{(n+1) / 2-\delta}$, where $n / 3 \leq \delta \leq(n-5) / 2$, by joining every vertex in $K_{\delta}$ to all other vertices and by adding a matching between all vertices in $K_{(n+1) / 2-\delta}$ and $(n+1) / 2-\delta$ vertices in $\bar{K}_{(n-1) / 2}$. It is easily seen that $G_{n}$ is 1-tough but not hamiltonian. A variation of the graph $G_{n}$, with $K_{\delta}$ replaced by $\bar{K}_{\delta}$ and $\delta=(n-5) / 2$, will be denoted by $G_{n}^{*}$.

\section{Pure relations for Hamilton cycles}

We begin with a pure algebraic relation between order $n$ and size $q$ insuring the existence of a Hamilton cycle based on the natural idea that if a sufficient number of edges are present in the graph then a Hamilton cycle will exist.

Theorem 1 (Erdös \& Gallai, 1959). Let $G$ be an arbitrary graph. If

$$
q \geq \frac{n^{2}-3 n+5}{2}
$$

then $G$ is hamiltonian.

Example for sharpness. To see that the size bound $\left(n^{2}-3 n+5\right) / 2$ in Theorem 1 is best possible, note that the graph formed by joining one vertex of $K_{n-1}$ to $K_{1}$, contains $\left(n^{2}-3 n+4\right) / 2$ edges and is not hamiltonian.

The next pure algebraic relation links the size $q$ and minimum degree $\delta$ insuring the existence of a Hamilton cycle. In view of Theorem 1, it seems a little surprising, providing, in fact, a contrary statement. 
Theorem 2 (Zh.G. Nikoghosyan, 2011). Let $G$ be an arbitrary graph. If

$$
q \leq \delta^{2}+\delta-1
$$

then $G$ is hamiltonian.

Example for sharpness. The bound $\delta^{2}+\delta-1$ in Theorem 2 can not be relaxed to $\delta^{2}+\delta$ since the graph $K_{1}+2 K_{\delta}$ consisting of two copies of $K_{\delta+1}$ and having exactly one vertex in common, has $\delta^{2}+\delta$ edges but is not hamiltonian.

The earliest sufficient condition for a graph to be hamiltonian is based on the order $n$ and minimum degree $\delta$ ensuring the existence of a Hamilton cycle with sufficient number of edges by keeping the minimum degree at a fairly high level.

Theorem 3 (Dirac, 1952). Let $G$ be an arbitrary graph. If

$$
\delta \geq \frac{n}{2}
$$

then $G$ is hamiltonian.

Example for sharpness: $2 K_{\delta}+K_{1}$.

The graph $2 K_{\delta}+K_{1}$ shows that the bound $n / 2$ in Theorem 3 can not be replaced by $(n-1) / 2$.

The minimum degree bound $n / 2$ in Theorem 3 can be slightly relaxed for graphs under additional 1-tough condition.

Theorem 4 (Jung, 1978). Let $G$ be a graph with $n \geq 11$ and $\tau \geq 1$. If

$$
\delta \geq \frac{n-4}{2}
$$

then $G$ is hamiltonian.

Examples for sharpness: Petersen graph; $K_{\delta, \delta+1} ; G_{n}^{*}$.

This bound $(n-4) / 2$ itself was lowered further to $(n-7) / 2$ under stronger conditions $n \geq 30$ and $\tau>1$.

Theorem 5 (Bauer et al., 1991a). Let $G$ be a graph with $n \geq 30$ and $\tau>1$. If

$$
\delta \geq \frac{n-7}{2}
$$

then $\mathrm{G}$ is hamiltonian.

Furthermore, the bound $n / 2$ was essentially lowered to $(n+\kappa) / 3$ (when $k<n / 2$ ) by incorporating connectivity $\kappa$ into the minimum degree bound. 
Theorem 6 (Zh.G. Nikoghosyan, 1981). Let $G$ be a graph with $\kappa \geq 2$. If

$$
\delta \geq \frac{n+\kappa}{3}
$$

then $\mathrm{G}$ is hamiltonian.

Examples for sharpness: $2 K_{\delta}+K_{1} ; H(1, \delta-\kappa+1, \delta, \kappa)(2 \leq \kappa<n / 2)$.

A short proof of Theorem 6 was given by Häggkvist (Häggkvist \& Nicoghossian, 1981).

The minimum degree bound $(n+\kappa) / 3$ in Theorem 6 was slightly lowered to $(n+\kappa-2) / 3$ for 1-tough graphs.

Theorem 7 (Bauer \& Schmeichel, 1991b). Let $G$ be a graph with $\tau \geq 1$. If

$$
\delta \geq \frac{n+\kappa-2}{3}
$$

then $G$ is hamiltonian.

Examples for sharpness: $K_{\delta, \delta+1} ; L_{\delta}$.

Another essential improvement of Dirac's bound $n / 2$ was established for 2-connected graphs under additional strong condition $\delta \geq \alpha$.

Theorem 8 (Nash-Williams, 1971). Let $G$ be a graph with $\kappa \geq 2$. If

$$
\delta \geq \max \left\{\frac{n+2}{3}, \alpha\right\}
$$

then $G$ is hamiltonian.

Examples for sharpness:

$(\lambda+1) K_{\delta-\lambda+1}+K_{\lambda}(\delta \geq 2 \lambda) ;(\lambda+2) K_{\delta-\lambda}+K_{\lambda+1}(\delta \geq 2 \lambda+1) ; H(\lambda, \lambda+1, \lambda+3, \lambda+2)$.

Theorem 8 was slightly improved by replacing the condition $\kappa \geq 2$ with a stronger condition $\tau \geq 1$.

Theorem 9 (Bigalke \& Jung, 1979). Let $G$ be a graph with $\tau \geq 1$. If

$$
\delta \geq \max \left\{\frac{n}{3}, \alpha-1\right\}
$$

then $G$ is hamiltonian.

Examples for sharpness: $K_{\delta, \delta+1}(n \geq 3) ; L_{\delta}(n \geq 7) ; K_{\delta, \delta+1}(n \geq 3)$. 
For $\lambda$ a positive integer, the bound $(n+2) / 3$ in Theorem 8 was essentially lowered under additional condition of the type $\delta \geq \alpha+\lambda$, including Theorem 8 as a special case.

Theorem 10 (Fraisse, 1986). Let $G$ be a graph, $\lambda$ a positive integer and

$$
\delta \geq \max \left\{\frac{n+2}{\lambda+2}+\lambda-1, \alpha+\lambda-1\right\} .
$$

If $\kappa \geq \lambda+1$ then $G$ is hamiltonian.

Examples for sharpness:

$(\lambda+1) K_{\delta-\lambda+1}+K_{\lambda}(\delta \geq 2 \lambda) ;(\lambda+2) K_{\delta-\lambda}+K_{\lambda+1}(\delta \geq 2 \lambda+1) ; H(\lambda, \lambda+1, \lambda+3, \lambda+2)$.

Later, Theorem 8 was essentially improved for 3-connected graphs by incorporating the connectivity $\kappa$ into the minimum degree bound.

Theorem 11 (Zh.G. Nikoghosyan, 1985a). Let $G$ be a graph with $\kappa \geq 3$. If

$$
\delta \geq \max \left\{\frac{n+2 \kappa}{4}, \alpha\right\}
$$

then $G$ is hamiltonian.

Examples for sharpness: $3 K_{2}+K_{2} ; 4 K_{2}+K_{3} ; H(1,2, \kappa+1, \kappa)$.

The graph $4 K_{2}+K_{3}$ shows that for $\kappa=3$ the minimum degree bound $(n+2 \kappa) / 4$ in Theorem 11 can not be replaced by $(n+2 \kappa-1) / 4$.

Finally, the bound $(n+2 \kappa) / 4$ in Theorem 11 was reduced to $(n+\kappa+3) / 4$ without any additional limitations providing a best possible result for each $\kappa \geq 3$.

Theorem 12 (Yamashita, 2008). Let $G$ be a graph with $\kappa \geq 3$. If

$$
\delta \geq \max \left\{\frac{n+\kappa+3}{4}, \alpha\right\}
$$

then $G$ is hamiltonian.

Examples for sharpness: $3 K_{\delta-1}+K_{2} ; H(2, n-3 \delta+3, \delta-1, \kappa) ; H(1,2, \kappa+1, \kappa)$.

The first pure relation between graph invariants involving connectivity $\kappa$ as a parameter was developed in 1972.

Theorem 13 (Chvátal and Erdös, 1972). Let G be an arbitrary graph. If

$$
\kappa \geq \alpha
$$

then $G$ is hamiltonian.

Example for sharpness: $K_{\delta, \delta+1}$. 


\section{Pure relations for dominating cycles}

In view of Theorem 2, the following upper size bound is reasonable for dominating cycles.

Conjecture 1. Let $G$ be a graph with $\kappa \geq 2$. If

$$
q \leq \frac{3\left(\delta^{2}+\delta-2\right)-1}{2}
$$

then each longest cycle in $G$ is a dominating cycle.

In 1971, it was proved that the minimum degree bound $(n+2) / 3$ insures the existence of dominating cycles.

Theorem 14 (Nash-Williams, 1971). Let $G$ be a graph with

$$
\delta \geq \frac{n+2}{3}
$$

If $\kappa \geq 2$ then each longest cycle in $G$ is a dominating cycle.

Examples for sharpness: $2 K_{3}+K_{1} ; 3 K_{\delta-1}+K_{2} ; H(1,2,4,3)$.

The graph $2 K_{3}+K_{1}$ shows that the connectivity condition $\kappa \geq 2$ in Theorem 14 can not be replaced by $\kappa \geq 1$. The second graph shows that the minimum degree condition $\delta \geq(n+2) / 3$ can not be replaced by $\delta \geq(n+1) / 3$ and the third graph shows that the conclusion "is a dominating cycle" can not be strengthened by replacing it with "is a Hamilton cycle".

The condition $\delta \geq(n+2) / 3$ in Theorem 14 can be slightly relaxed under stronger 1 -tough condition instead of $\kappa \geq 2$.

Theorem 15 (Bigalke \& Jung, 1979). Let $G$ be a graph with $\tau \geq 1$. If

$$
\delta \geq \frac{n}{3}
$$

then each longest cycle in $G$ is a dominating cycle.

Examples for sharpness: $2(\kappa+1) K_{2}+\kappa K_{1} ; L_{3} ; G_{n}^{*}$.

The bound $(n+2) / 3$ in Theorem 14 can be lowered to $(n+2 \kappa) / 4$ by incorporating $\kappa$ into the minimum degree bound.

Theorem 16 (Lu et al., 2005). Let $G$ be graph with $\kappa \geq 3$. If

$$
\delta \geq \frac{n+2 \kappa}{4}
$$

then each longest cycle in $G$ is a dominating cycle. 
Examples for sharpness: $3 K_{2}+K_{2} ; 4 K_{2}+K_{3} ; H(1,2, \kappa+1, \kappa)$.

The graph $4 K_{2}+K_{3}$ shows that for $\kappa=3$ the minimum degree bound $(n+2 \kappa) / 4$ in Theorem 16 can not be replaced by $(n+2 \kappa-1) / 4$.

In 2008 , the bound $(n+2 \kappa) / 4$ itself was essentially reduced to $(n+\kappa+3) / 4$ without any additional limitations, providing a best possible result for each $\kappa \geq 3$.

Theorem 17 (Yamashita, 2008). Let $G$ be graph with $\kappa \geq 3$. If

$$
\delta \geq \frac{n+\kappa+3}{4}
$$

then each longest cycle in $G$ is a dominating cycle.

Examples for sharpness: $3 K_{\delta-1}+K_{2} ; H(2, n-3 \delta+3, \delta-1, \kappa) ; H(1,2, \kappa+1, \kappa)$.

\section{Pure relations for $C D_{\lambda}$-cycles}

In 1990, the exact analog of Theorems 3 and 14 was established In terms of generalized $C D_{3}$ cycles.

Theorem 18 (Jung, 1990). Let $G$ be a graph with

$$
\delta \geq \frac{n+6}{4} .
$$

If $\kappa \geq 3$ then each longest cycle in $G$ is a $C D_{3}$-cycle.

Examples for sharpness:

$\lambda K_{\lambda+1}+K_{\lambda-1}(\lambda \geq 2) ;(\lambda+1) K_{\delta-\lambda+1}+K_{\lambda}(\lambda \geq 1) ; H(\lambda-1, \lambda, \lambda+2, \lambda+1)(\lambda \geq 2)$.

In 2009, a common generalization of Theorems 3, 14 and 18 was proved by covering $C D_{\lambda}$ cycles for each integer $\lambda \geq 1$.

Theorem 19 (Zh.G. Nikoghosyan, 2009a). Let $G$ be a graph, $\lambda$ a positive integer and

$$
\delta \geq \frac{n+2}{\lambda+1}+\lambda-2 .
$$

Then each longest cycle in $G$ is a $C D_{\min \{\lambda, \delta-\lambda+1\}}$-cycle.

Examples for sharpness:

$\lambda K_{\lambda+1} K_{\lambda-1}(\lambda \geq 2) ;(\lambda+1) K_{\delta-\lambda+1}+K_{\lambda}(\lambda \geq 1) ; H(\lambda-1, \lambda, \lambda+2, \lambda+1)(\lambda \geq 2)$.

In (Zh.G. Nikoghosyan, 2009a), an analogous generalization has been conjectured in terms of $P D_{\lambda}$-cycles.

Conjecture 1 (Zh.G. Nikoghosyan, 2009a). Let $G$ be a graph, $\lambda$ a positive integer and $\kappa \geq \lambda$. If 


$$
\delta \geq \frac{n+2}{\lambda+1}+\lambda-2
$$

then each longest cycle in $G$ is a $P D_{\min \{\lambda-1, \delta-\lambda\}}$-cycle.

In view of Theorems 6 and 17, the next generalization seems reasonable.

Conjecture 2 (Yamashita, 2008). Let $G$ be graph, $\lambda$ an integer and $\kappa \geq \lambda \geq 2$. If

$$
\delta \geq \frac{n+\kappa+\lambda(\lambda-2)}{\lambda+1}
$$

then each longest cycle in $G$ is a $P D_{\lambda-2}$ and $C D_{\lambda-1}$-cycle.

\section{Pure relations for long cycles}

The earliest and simplest hamiltonian result links the circumference $\mathrm{c}$ and minimum degree $\delta$.

Theorem 20 (Dirac, 1952). In every graph,

$$
c \geq \delta+1 .
$$

Example for sharpness: Join two copies of $K_{\delta+1}$ by an edge.

For $C$ a longest cycle in a graph $G$, a lower bound for $|C|$ was developed based on the minimum degree $\delta$ and $\bar{p}$ - the length of a longest path in $G \backslash C$.

Theorem 21 (Zh.G. Nikoghosyan, 1998). Let $G$ be a graph and $C$ a longest cycle in $G$. Then

$$
|C| \geq(\bar{p}+2)(\delta-\bar{p}) \text {. }
$$

Example for sharpness: $(\kappa+1) K_{\delta-\kappa+1}+K_{\kappa}$.

The next similar bound is based On the minimum degree $\delta$ and $\bar{c}$ - the length of a longest cycle in $G \backslash C$.

Theorem 22 (Zh.G. Nikoghosyan, 2000a). Let $G$ be a graph and $C$ a longest cycle in $G$. Then

$$
|C| \geq(\bar{c}+1)(\delta-\bar{c}+1)
$$

Example for sharpness: $(\kappa+1) K_{\delta-\kappa+1}+K_{\kappa}$.

In 2000, Theorem 22 was improved involving connectivity $\kappa$ as a parameter combined with $\bar{c}$ and $\delta$.

Theorem 23 (Zh.G. Nikoghosyan, 2000b). Let $G$ be a graph with $\kappa \geq 2$ and $C$ a longest cycle in $G$. If $\bar{c} \geq \kappa$ then 


$$
|C| \geq \frac{(\bar{c}+1) \kappa}{\bar{c}+\kappa+1}(\delta+2) .
$$

Otherwise,

$$
|C| \geq \frac{(\bar{c}+1) \bar{c}}{2 \bar{c}+1}(\delta+2)
$$

Example for sharpness: $(\kappa+1) K_{\delta-\kappa+1}+K_{\kappa}$.

In view of Theorem 23, the following seems reasonable for $P D_{\lambda}$-cycles.

Conjecture 3 (Zh.G. Nikoghosyan, 2009a). Let $G$ be a graph with $\kappa \geq 2$ and $C$ a longest cycle in $G$. If $\bar{p} \geq \kappa-1$ then

$$
|C| \geq \frac{(\bar{p}+2) \kappa}{\bar{p}+\kappa+2}(\delta+2)
$$

Otherwise,

$$
|C| \geq \frac{(\bar{p}+2) \bar{p}}{2 \bar{p}+2}(\delta+2) .
$$

\section{Pure relations for Hamilton cycles and long cycles}

The following direct generalization includes Theorem 3 as a special case.

Theorem 24 (Alon, 1986). Let $G$ be a graph and $\lambda$ a positive integer. If $\delta \geq n /(\lambda+1)$ then

$$
c \geq \frac{n}{\lambda}
$$

Examples for sharpness: $(\lambda+1) K_{\lambda}+K_{1} ; \lambda K_{\lambda+1}$.

In 1952, a relationship was established linking the minimum degree $\delta$, circumference $c$ and Hamilton cycles for 2-connexted graphs.

Theorem 25 (Dirac, 1952). Let $G$ be a graph with $\kappa \geq 2$. Then

$$
c \geq \min \{n, 2 \delta\} .
$$

Examples for sharpness:

$(\lambda+1) K_{\lambda+1}+K_{\lambda}(\lambda \geq 1) ;(\lambda+3) K_{\lambda-1}+K_{\lambda+2}(\lambda \geq 2) ;(\lambda+2) K_{\lambda}+K_{\lambda+1}(\lambda \geq 1)$.

For 1-tough graphs the bound $2 \delta$ in Theorem 25 was slightly enlarged.

Theorem 26 (Bauer and Schmeichel, 1986). Let $G$ be a graph with $\tau \geq 1$. Then 


$$
c \geq \min \{n, 2 \delta+2\}
$$

Examples for sharpness: $K_{\delta, \delta+1} ; L_{2}$.

The first essential improvement of Theorem 25 was achieved by incorporating connectivity $\kappa$ into the relation without any essential limitation.

Theorem 27 (Zh.G. Nikoghosyan, 1981). Let $G$ be a graph with $\kappa \geq 3$. Then

$$
c \geq \min \{n, 3 \delta-\kappa\} .
$$

Examples for sharpness: $3 K_{\delta-1}+K_{2} ; H(1, \delta-\kappa+1, \delta, \kappa)$.

In (Voss \& Zuluaga, 1977), it was proved that the bound $\min \{n, 2 \delta\}$ in Theorem 25 can be essentially enlarged under additional condition $\delta \geq \alpha$ combined with $\kappa \geq 3$.

Theorem 28 (Voss and Zuluaga, 1977). Let $G$ be a graph with $\kappa \geq 3$. If $\delta \geq \alpha$ then

$$
c \geq \min \{n, 3 \delta-3\}
$$

Examples for sharpness: $(\lambda+2) K_{\lambda+2}+K_{\lambda+1} ;(\lambda+4) K_{\lambda}+K_{\lambda+3} ;(\lambda+3) K_{\lambda+1}+K_{\lambda+2}$.

In 2009, a direct generalization was established for each positive integer $\lambda$, including Theorem 28 as a special case $(\lambda=1)$.

Theorem 29 (Zh.G. Nikoghosyan, 2009a). Let $G$ be a graph and $\lambda$ a positive integer. If $\kappa \geq \lambda+2$ and $\delta \geq \alpha+\lambda-1$ then

$$
c \geq \min \{n,(\lambda+2)(\delta-\lambda)\} .
$$

Examples for sharpness: $(\lambda+2) K_{\lambda+2}+K_{\lambda+1} ;(\lambda+4) K_{\lambda}+K_{\lambda+3} ;(\lambda+3) K_{\lambda+1}+K_{\lambda+2}$.

In 1985 , the bound $3 \delta-\kappa$ in Theorem 27 was enlarged to $4 \delta-2 \kappa$ under additional condition $\delta \geq \alpha$ combined with $\kappa \geq 4$.

Theorem 30 (Zh.G. Nikoghosyan, 1985b). Let $G$ be a graph with $\kappa \geq 4$ and $\delta \geq \alpha$. Then

$$
c \geq \min \{n, 4 \delta-2 \kappa\} .
$$

Examples for sharpness: $4 K_{2}+K_{3} ; H(1, n-2 \delta, \delta, \kappa) ; 5 K_{2}+K_{4}$.

The bound $4 \delta-2 \kappa$ in Theorem 30 is sharp for $\kappa=4$.

Furthermore, the bound $4 \delta-2 \kappa$ in Theorem 30 was essentially improved to $4 \delta-\kappa-4$ without any additional limitations providing a best possible result for each $\kappa \geq 4$.

Theorem 31 (M.Zh. Nikoghosyan \& Zh.G. Nikoghosyan, 2011). Let $G$ be a graph with $\kappa \geq 4$ and $\delta \geq \alpha$. Then 


$$
c \geq \min \{n, 4 \delta-\kappa-4\} .
$$

Examples for sharpness: $4 K_{\delta-2}+K_{3} ; H(1,2, \kappa+1, \kappa) ; H(2, n-3 \delta+3, \delta-1, \kappa)$.

The next theorem provides a lower bound for the circumference in terms of $n, \delta$ and $\alpha$ under the hypothesis of Theorem 14.

Theorem 32 (Bauer et al., 1990b). Let $G$ be a graph with $\kappa \geq 2$. If $\delta \geq(n+2) / 3$ then

$$
c \geq \min \{n, n+\delta-\alpha\}
$$

Examples for sharpness: $2 K_{\delta}+K_{1} ; 3 K_{\delta-1}+K_{2} ; K_{2 \delta-2, \delta}$.

An analogous bound was established for 1-tough graphs.

Theorem 33 (Bauer et al., 1988). Let $G$ be a graph with $\tau \geq 1$. If $\delta \geq n / 3$ then

$$
c \geq \min \{n, n+\delta-\alpha+1\} .
$$

Examples for sharpness: $K_{\delta, \delta+1} ; L_{\delta} ; G_{n}^{*}$.

\section{Pure relations for dominating cycles and long cycles}

The exact analog of Theorem 25 for dominating cycles can be formulated as follows.

Theorem 34 (Voss \& Zuluaga, 1977). Let $G$ be a graph with $\kappa \geq 3$. Then either

$$
c \geq 3 \delta-3
$$

or each longest cycle in $G$ is a dominating cycle.

Examples for sharpness:

$$
(\lambda+1) K_{\lambda+1}+K_{\lambda}(\lambda \geq 1) ;(\lambda+3) K_{\lambda+1}+K_{\lambda+2}(\lambda \geq 2) ;(\lambda+2) K_{\lambda}+K_{\lambda+1}(\lambda \geq 1) .
$$

The bound $3 \delta-3$ in Theorem 34 was enlarged to $4 \delta-2 \kappa$ by incorporating connectivity $\kappa$ into the bound.

Theorem 35 (Zh.G. Nikoghosyan, 2009b). Let $G$ be a graph with $\kappa \geq 4$. Then either

$$
c \geq 4 \delta-2 \kappa
$$

or $G$ has a dominating cycle.

Examples for sharpness: $4 K_{2}+K_{3} ; 5 K_{2}+K_{4} ; H(1, n-2 \delta, \delta, \kappa)$.

Theorem 35 is sharp only for $\kappa=4$ as can be seen from $5 K_{2}+K_{4}$. Further, the bound $4 \delta-2 \kappa$ in Theorem 35 was essentially improved to $4 \delta-\kappa-4$ without any limitation providing a sharp bound for each $\kappa \geq 4$. 
Theorem 36 (M.Zh. Nikoghosyan \& Zh.G. Nikoghosyan, 2011). Let G be a graph with $\kappa \geq 4$. Then either

$$
c \geq 4 \delta-\kappa-4
$$

or each longest cycle in $G$ is a dominating cycle.

Examples for sharpness: $4 K_{\delta-2}+K_{3} ; H(2, \delta-\kappa+1, \delta-1, \kappa) ; H(1,2, \kappa+1, \kappa)$.

\section{Pure relations for $C D_{\lambda}$-cycles and long cycles}

TThe following theorem can be considered as a common generalization of Theorems 25 and 34 by covering $C D_{\lambda}$-cycles for all $\lambda \geq 1$ including Hamilton and dominating cycles as special cases.

Theorem 37 (Zh.G. Nikoghosyan, 2009a). Let $G$ be a graph and $\lambda$ a positive integer. If $\kappa \geq \lambda+1$ then either

$$
c \geq(\lambda+1)(\delta-\lambda+1)
$$

or each longest cycle in $G$ is a $C D_{\min \{\lambda, \delta-\lambda\}}$-cycle.

Examples for sharpness:

$(\lambda+1) K_{\lambda+1}+K_{\lambda}(\lambda \geq 1) ;(\lambda+3) K_{\lambda-1}+K_{\lambda+2}(\lambda \geq 2) ;(\lambda+2) K_{\lambda}+K_{\lambda+1}(\lambda \geq 1)$.

In (Zh.G. Nikoghosyan, 2009a), another version of Theorem 37 was conjectured in terms of $P D_{\lambda}$-cycles, instead of $C D_{\lambda}$-cycles.

Conjecture 4 (Zh.G. Nikoghosyan, 2009a). Let $G$ be a graph and $\lambda$ a positive integer. If $\kappa \geq \lambda+1$ then either

$$
c \geq(\lambda+1)(\delta-\lambda+1)
$$

or each longest cycle in $G$ is a $P D_{\min \{\lambda-1, \delta-\lambda-1\}}$-cycle.

In view of Theorems 27 and 36, the following common generalization seems quite reasonable.

Conjecture 5. Let $G$ be a graph and $\lambda \geq 2$ an integer. If $\kappa \geq \lambda+1$ then either

$$
c \geq(\lambda+1) \delta-\kappa-(\lambda+1)(\lambda-2)
$$

or each longest cycle in $G$ is a $P D_{\lambda-2}$ and $C D_{\lambda-1}$-cycle.

\section{Proofs of theorems 6, 21 and 27}

In proofs of theorems and lemmas, the end of the proof is marked by $\square$. In proofs of claims, the end of the proof is marked by $\Delta$. 
Proof of Theorem 27 (Mosesyan et al., 2009). Let $G$ be a 3-connected graph and $S$ a minimum cut-set in $G$. Choose a longest cycle $C$ in $G$ so as to maximize $|V(C) \cap S|$. The result holds immediately if $|C| \geq 3 \delta-3$, since $3 \delta-3 \geq 3 \delta-\kappa$. Otherwise, by Theorem 34, $C$ is a dominating cycle. Assume first that $S \not V(C)$ and let $v \in S \backslash V(C)$. Since $\rightarrow C$ is dominating, $N(v) \subseteq V(C)$. Let $\xi_{1}, \ldots, \xi_{t}$ be the elements of $N(v)$, occurring on $\vec{C}$ in a consecutive order. Put

$$
M_{1}=\left\{\xi_{i} \mid V\left(\xi_{i}^{+} \vec{C} \xi_{i+1}^{-}\right) \cap S \neq \varnothing\right\}, \quad M_{2}=N(v) \backslash M_{1} .
$$

Since $v \in S$, we have

$$
\left|M_{1}\right| \leq \kappa-1, \quad\left|M_{2}\right|=|N(v)|-\left|M_{1}\right| \geq \delta-\kappa+1 .
$$

Further, since $C$ is extreme and $|V(C) \cap S|$ is maximum,

$$
N(v) \cap N^{+}(v) \cap M_{2}^{++}=\varnothing .
$$

Hence

$$
\begin{aligned}
& |C| \geq|N(v)|+\left|N^{+}(v)\right|+\left|M_{2}^{++}\right| \\
& =2|N(v)|+\left|M_{2}\right| \geq 3 \delta-\kappa+1 .
\end{aligned}
$$

Now assume that $S \subseteq V(C)$. Let $H_{1}, \ldots, H_{h}$ be the connected components of $G \backslash S$. If $V(G \backslash C)=\varnothing$ then $|C|=n$ and we are done. Let $x \in V(G \backslash C)$. Assume without loss of generality that $x \in V\left(H_{1}\right)$. Since $C$ is dominating, $N(x) \subseteq V(C)$. Put $Y_{1}=N(x) \cup N^{+}(x)$. Clearly $\left|Y_{1}\right| \geq 2 \delta$ and to prove that $|C| \geq 3 \delta-\kappa$, it remains to find a subset $Y_{2}$ in $V(C)$ such that $Y_{1} \cap Y_{2}=\varnothing$ and $\left|Y_{2}\right| \geq \delta-\kappa$. Abbreviate, $V_{1}=V\left(H_{1}\right) \cup S$. Suppose first that $Y_{1} \subseteq V_{1}$. If $V\left(H_{2}\right) \subseteq V(C)$ then $Y_{2}=V\left(H_{2}\right)$ since $\left|V\left(H_{2}\right)\right| \geq \delta-\kappa+1$. Otherwise, there exist $y \in V\left(H_{2} \backslash C\right)$. Since $C$ is dominating, $N(y) \subseteq V(C)$ and we can take $Y_{2}=N(y) \backslash S$. Now let $Y_{1} \nsubseteq V_{1}$. Assume without loss of generality that $Y_{1} \cap V\left(H_{2}\right) \neq \varnothing$. Since $N(x) \subseteq V_{1}$, we have $N^{+}(x) \cap V\left(H_{2}\right) \neq \varnothing$. Let $z \in N^{+}(x) \cap V\left(H_{2}\right)$. If $N(z) \subseteq V(C)$ then take $Y_{2}=N(z) \backslash S$, since $N^{+}(x)$ is an independent set of vertices (by standard arguments) and therefore, $N(z) \cap N^{+}(x)=\varnothing$. Otherwise, choose $w \in N(z) \backslash V(C)$. Clearly

$$
N(w) \subseteq V(C), w \in V\left(H_{2}\right), N(w) \cap N^{+}(x)=\{z\}
$$

Then by taking

$$
Y_{2}=(N(w) \backslash\{z\}) \backslash\left(S \backslash\left\{z^{-}\right\}\right)
$$


we complete the proof of Theorem 27.

Proof of Theorem 6 (Mosesyan et al., 2009). Let $G$ be a 2-connected graph with $\delta \geq(n+\kappa) / 3$ and let $S$ be a minimum cut-set in $G$. Since $\delta \geq(n+\kappa) / 3 \geq(n+2) / 3$, by Theorem 14, every longest cycle in $G$ is a dominating cycle. As in proof of Theorem 27, we can show that either $G$ is hamiltonian or $c \geq 3 \delta-\kappa$. Since $n \leq 3 \delta-\kappa$ (by the hypothesis), it follows from $c \geq 3 \delta-\kappa$ that $c=3 \delta-\kappa=n$. So, in any case, $G$ is hamiltonian.

To prove Theorem 21, we need some special definitions. Let $G$ be a graph, $C$ a longest cycle in $G$ and $\vec{M}$ a longest path (or a cycle) in $G \backslash C$. Further, let $u_{1}, \ldots, u_{m}$ be the elements of $V(M)$ occurring on $\vec{M}$ in a consecutive order.

Definition $1\left\{M_{C}\right.$-spreading; $\left.\vec{\Upsilon}(u) ; \dot{u} ; \ddot{u}\right\}$. An $M_{C}$-spreading $\Upsilon$ is a family of pairwise disjoint paths $\vec{\Upsilon}\left(u_{1}\right), \ldots, \vec{\Upsilon}\left(u_{m}\right)$ in $G \backslash C$ with $\vec{\Upsilon}\left(u_{i}\right)=u_{i} \vec{\Upsilon}\left(u_{i}\right) \ddot{u}_{i} \quad(i=1, \ldots, m)$. If $u \neq \ddot{u}$ for some $\vec{\Upsilon}(u)$, then we use $\dot{u}$ to denote the successor of $u$ along $\vec{\Upsilon}(u)$.

Definition $2\left\{\Phi_{u} ; \varphi_{u} ; \Psi_{u} ; \psi_{u}\right\}$. Let $\Upsilon$ be any $M_{C}$-spreading. For each $u \in V(M)$, put

$$
\begin{gathered}
\Phi_{u}=N(\ddot{u}) \cap V(\Upsilon), \quad \varphi_{u}=\left|\Phi_{u}\right|, \\
\Psi_{u}=N(\ddot{u}) \cap V(H), \quad \Psi_{u}=\left|\Psi_{u}\right| .
\end{gathered}
$$

Definition $3\left\{U_{0} ; \bar{U}_{0} ; U_{1} ; U^{*}\right\}$. For $\Upsilon$ an $M_{C}$-spreading, put

$$
\begin{aligned}
& U_{0}=\{u \in V(M) \mid u=\ddot{u}\}, \quad \bar{U}_{0}=V(M) \backslash U_{0}, \\
& U^{*}=\left\{u \in \bar{U}_{0} \mid \Phi_{u} \subseteq V(\Upsilon(u))\right\}, \quad U_{1}=V(M) \backslash\left(U_{0} \cup U^{*}\right) .
\end{aligned}
$$

Definition $4\left\{\left(U_{0}\right)\right.$-minimal $M_{C}$-spreading $\}$. An $M_{C}$-spreading $\Upsilon$ is said to be $\left(U_{0}\right)$ minimal, if it is chosen such that $\left|U_{0}\right|$ is minimum.

Definition $5\left\{B_{u} ; B_{u}^{*} ; b_{u} ; b_{u}^{*}\right\}$. For $\Upsilon$ an $M_{C}$-spreading and $u \in V(M)$, set

$$
B_{u}=\left\{v \in U_{0} \mid v \dot{u} \in E\right\}, \quad b_{u}=\left|B_{u}\right|
$$

Further, for each $u \in U_{0}$, set

$$
B_{u}^{*}=\left\{v \in \bar{U}_{0} \mid u \dot{v} \in E\right\}, \quad b_{u}^{*}=\left|B_{u}^{*}\right| .
$$


Lemma 1. Let $C$ be a longest cycle in a graph $G$ and $M$ a path in $G \backslash C$. Let $\vec{L}_{1}, \ldots, \vec{L}_{r}$ be vertex disjoint paths in $G \backslash C$ with $\vec{L}_{i}=v_{i} \vec{L}_{i} w_{i}(i=1, \ldots, r)$ having only $v_{1}, \ldots, v_{r}$ in common with $M$ and let. $Z_{i}=N\left(w_{i}\right) \cap V(C)(i=1, \ldots, r)$.. Then

$$
|C| \geq \sum_{i=1}^{r}\left|Z_{i}\right|+\left|\bigcup_{i=1}^{r} Z_{i}\right|
$$

Proof. We can assume without loss of generality that $v_{i}=w_{i}(i=1, \ldots, r)$, since otherwise, we can use the same arguments. If $\bigcup_{i=1}^{r} Z_{i}=\varnothing$, then there is nothing to prove. Let $\bigcup_{i=1}^{r} Z_{i} \neq \varnothing$ and let $\xi_{1}, \ldots, \xi_{t}$ be the elements of $\bigcup_{i=1}^{r} Z_{i}$ occurring on $\vec{C}$ in a consecutive order. Set

$$
F_{i}=N\left(\xi_{i}\right) \cap\left\{w_{1}, \ldots, w_{r}\right\} \quad(i=1, \ldots, t) .
$$

Suppose first that $t=1$. If $\left|F_{1}\right|=1$ then $\sum_{i=1}^{r}\left|Z_{i}\right|=\left|\bigcup_{i=1}^{r} Z_{i}\right|=1$ and the result follows from $|C| \geq 2$ immediately. If $\left|F_{1}\right| \geq 2$, then choosing a largest segment $u \vec{M} v$ on $M$ with $u, v \in F_{1}$, we get a cycle $C^{\prime}=\xi_{1} u \vec{M} v \xi_{1}$ satisfying

$$
|C| \geq\left|C^{\prime}\right| \geq \sum_{i=1}^{r}\left|Z_{1}\right|+1=\sum_{i=1}^{r}\left|Z_{1}\right|+\left|\bigcup_{i=1}^{r} Z_{i}\right| .
$$

Now assume $t \geq 2$. Put

$$
f\left(\xi_{i}\right)=\left|\xi_{i} \vec{C} \xi_{i+1}\right| \quad(i=1,2, \ldots, t)
$$

where $\xi_{i+1}=\xi_{1}$. Then it is easy to see that

$$
|C|=\sum_{i=1}^{t} f\left(\xi_{i}\right), \quad \sum_{i=1}^{t}\left|F_{i}\right|=\sum_{i=1}^{r}\left|Z_{i}\right|, \quad t=\left|\bigcup_{i=1}^{r} Z_{i}\right| .
$$

For each $i \in\{1, \ldots, t\}$, let $x_{i} \vec{M} y_{i}$ be the largest segment on $\vec{M}$ with $x_{i}, y_{i} \in F_{i} \cup F_{i+1}$ (indices $\bmod t$ ). Now we need to show that

$f\left(\xi_{i}\right) \geq\left(\left|F_{i}\right|+\left|F_{i+1}\right|+2\right) / 2$. Indeed, if $x_{i} \in F_{i}$ and $y_{i} \in F_{i+1}$, then $f\left(\xi_{i}\right) \geq\left|\xi_{i} x_{i} \vec{M} y_{i} \xi_{i+1}\right|$ since $C$ is extreme. It means that 


$$
f\left(\xi_{i}\right) \geq \max \left\{\left|F_{i}\right|,\left|F_{i+1}\right|\right\}+1 \geq \frac{1}{2}\left(\left|F_{i}\right|+\left|F_{i+1}\right|+2\right) .
$$

The same inequality holds from $f\left(\xi_{i}\right) \geq\left|\xi_{i} y_{i} \overleftarrow{M} x_{i} \xi_{i+1}\right|$ if $x_{i} \in F_{i+1}$ and $y_{i} \in F_{i}$, by a similar argument. Now suppose that either $x_{i}, y_{i} \in F_{i}$ or $x_{i}, y_{i} \in F_{i+1}$. Assume without loss of generality that $x_{i}, y_{i} \in F_{i}$. In addition, we have $x_{i}, y_{i} \notin F_{i+1}$, since otherwise we are in the previous case. Let $x_{i}^{\prime} \vec{M} y_{i}^{\prime}$ be the largest segment on $\vec{M}$ with $x_{i}^{\prime}, y_{i}^{\prime} \in F_{i+1}$. If $\left|x_{i} \vec{M} x_{i}^{\prime}\right| \geq\left(\left|F_{i}\right|-\left|F_{i+1}\right|\right) / 2$, then $f\left(\xi_{i}\right) \geq\left|\xi_{i} x_{i} \vec{M} y_{i}^{\prime} \xi_{i+1}\right|$ and hence

$$
f\left(\xi_{i}\right) \geq \frac{1}{2}\left(\left|F_{i}\right|-\left|F_{i+1}\right|\right)+\left|F_{i+1}\right|+1=\frac{1}{2}\left(\left|F_{i}\right|+\left|F_{i+1}\right|+2\right) .
$$

Finally, if $\left|x_{i} \vec{M} x_{i}^{\prime}\right| \leq\left(\left|F_{i}\right|-\left|F_{i+1}\right|-1\right) / 2$, then

$$
\begin{aligned}
& f\left(\xi_{i}\right) \geq\left|\xi_{i} y_{i} \overleftarrow{M} x_{i}^{\prime} \xi_{i+1}\right|=\left|x_{i}^{\prime} \vec{M} y_{i}\right|+2=\left|x_{i} \vec{M} y_{i}\right|-\left|x_{i} \vec{M} x_{i}^{\prime}\right|+2 \\
& \geq\left|F_{i}\right|-1-\frac{1}{2}\left(\left|F_{i}\right|-\left|F_{i+1}\right|-1\right)+2>\frac{1}{2}\left(\left|F_{i}\right|+\left|F_{i+1}\right|+2\right)
\end{aligned}
$$

So, $f\left(\xi_{i}\right) \geq\left(\left|F_{i}\right|+\left|F_{i+1}\right|+2\right) / 2 \quad(i=1, \ldots, t)$ in any case, implying that

$$
\sum_{i=1}^{t} f\left(\xi_{i}\right) \geq \sum_{i=1}^{t} \frac{1}{2}\left(\left|F_{i}\right|+\left|F_{i+1}\right|+2\right)=\sum_{i=1}^{t}\left|F_{i}\right|+t
$$

and the result follows from (1).

Lemma 2. Let $C$ be a longest cycle in a graph $G$ and $M$ a longest cycle in $G \backslash C$ with a $U_{0}$-minimal $M_{C}$-spreading $\Upsilon$. Then for each $u \in U_{1},|M| \geq \varphi_{u}+b_{u}+1$.

Proof. Let $u \in U_{1}$. For each $x \in V(M)$, put

$A_{u}(x)=\left(\Phi_{u} \cup B_{u}\right) \cap V(\Upsilon(x))$. By the definition,

$$
\left|\Phi_{u} \cup B_{u}\right|=\sum_{x \in V(M)}\left|A_{u}(x)\right|
$$

If $A_{u}(x) \neq \varnothing$ for some $x \in V(M)$, then we choose a vertex $\rho_{u}(x)$ in $A_{u}(x)$ such that $\left|x \vec{\Upsilon}(x) \rho_{u}(x)\right|$ is maximum. By the definition, $\rho_{u}(u)=(\ddot{u})^{-}$. Put $\bar{\rho}_{u}(x)=\ddot{u}$ if $\rho_{u}(x) \in \Phi_{u}$, and $\bar{\rho}_{u}(x)=\dot{u}$ if $\rho_{u}(x) \in B_{u} \backslash \Phi_{u}$. Clearly $\bar{\rho}_{u}(u)=\ddot{u}$.

Let $\Lambda_{u}=\left\{x \in V(M) \mid A_{u}(x) \neq \varnothing\right\}$. Further, for each distinct $x, y \in \Lambda_{u}$, put $\Lambda_{u}(x, y)=x \dot{u} y$ if either $x=u, y \in U_{0}$ or $y=u, x \in U_{0}$. Otherwise, 


$$
\Lambda_{u}(x, y)=x \vec{\Upsilon}(x) \rho_{u}(x) \bar{\rho}_{u}(x) \Upsilon(u) \bar{\rho}_{u}(y) \rho_{u}(y) \overleftarrow{\Upsilon}(y) y
$$

Let $\xi_{1}, \ldots, \xi_{f}$ be the elements of $\Lambda_{u}$, occurring on $\vec{M}$ in a consecutive order with $\xi_{1}=u$. For each integer $i(1 \leq i \leq f)$, set

$$
M_{i}=\xi_{i} \vec{M} \xi_{i+1}, \quad \omega_{i}=\left|A_{u}\left(\xi_{i}\right)\right|+\left|A_{u}\left(\xi_{i+1}\right)\right| \quad(\text { indices } \bmod f)
$$

Claim 1. $\sum_{i=1}^{f}\left|M_{i}\right| \geq \sum_{i=1}^{f} \omega_{i}$.

Proof. Since $M$ is extreme, for each $i \in\{2, \ldots, f-1\}$,

$$
\left|M_{i}\right| \geq\left|\Lambda_{u}\left(\xi_{i}, \xi_{i+1}\right)\right| \geq\left|A_{u}\left(\xi_{i}\right)\right|+\left|A_{u}\left(\xi_{i+1}\right)\right|=\omega_{i} .
$$

If $\Phi_{u} \cap V\left(\Upsilon\left(\xi_{2}\right)\right) \neq \varnothing$ and $\Phi_{u} \cap V\left(\Upsilon\left(\xi_{f}\right)\right) \neq \varnothing$, then the inequality $\left|M_{i}\right| \geq \omega_{i}(i=1, f)$ holds as in previous case and we are done. Now let $\Phi_{u} \cap V\left(\Upsilon\left(\xi_{2}\right)\right)=\varnothing$ and $\Phi_{u} \cap V\left(\Upsilon\left(\xi_{f}\right)\right)=\varnothing$. It means that $A_{u}\left(\xi_{2}\right)=\left\{\xi_{2}\right\}$ and therefore, $\left|M_{1}\right| \geq 2=\left|A_{u}\left(\xi_{2}\right)\right|+1=\omega_{1}-\left|A_{u}(u)\right|+1$. Analogously, $\left|M_{f}\right| \geq \omega_{f}-\left|A_{u}(u)\right|+1$. By the definition of $\Lambda_{u}, \dot{u} \xi_{2} \in E$ and $\dot{u} \xi_{f} \in E$. Since $u \in U_{1}$, we have $\Phi_{u} \cap V\left(\Upsilon\left(\xi_{s}\right)\right) \neq \varnothing$ for some $3 \leq s \leq f-1$. Then we can choose $i, j$ such that $2 \leq i \leq s-1$ and $s \leq j \leq f-1$ with $\left|M_{i}\right| \geq \omega_{i}+\left|A_{u}(u)\right|-1$ and $\left|M_{j}\right| \geq \omega_{j}+\left|A_{u}(u)\right|-1$, and the result follows. Finally, because of the symmetry, we can suppose that $\Phi_{u} \cap V\left(\Upsilon\left(\xi_{2}\right)\right)=\varnothing \quad$ and $\quad \Phi_{u} \cap V\left(\Upsilon\left(\xi_{f}\right)\right) \neq \varnothing$. Clearly $\quad\left|M_{1}\right| \geq \omega_{1}-\left|A_{u}(u)\right|+1$. By the definition of $\Lambda_{u}, \dot{u} \xi_{2} \in E$. Then we can choose $i \in\{2, \ldots, f-1\}$ such that $\left|M_{i}\right| \geq \omega_{i}+\left|A_{u}(u)\right|-1$ and again the result follows. $\Delta$

Claim 2. If $|\Upsilon(u)| \geq 2$ then $\Phi_{u} \cap U_{0}=\varnothing$.

Proof. Suppose to the contrary and let $v \in \Phi_{u} \cap U_{0}$. Then replacing $\Upsilon(u)$ and $\Upsilon(v)$ by $u \vec{\Upsilon}(u)(\ddot{u})^{-}$and $v \ddot{u}$, respectively, we can form a new $M_{H}$-spreading, contradicting the $\left(U_{0}\right)$-minimality of $\Upsilon . \Delta$

By (2) and Claim 1,

$$
\begin{aligned}
& |M|=\sum_{i=1}^{f}\left|M_{i}\right| \geq \sum_{i=1}^{f} \omega_{i}=\sum_{i=1}^{f}\left(\left|A_{u}\left(\xi_{i}\right)\right|+\left|A_{u}\left(\xi_{i+1}\right)\right|\right) \\
& =2 \sum_{i=1}^{f}\left|A_{u}\left(\xi_{i}\right)\right|=2 \sum_{x \in V(M)}\left|A_{u}(x)\right|=2\left|\Phi_{u} \cup B_{u}\right| .
\end{aligned}
$$


If $|\Upsilon(u)| \geq 2$ then by Claim 2, $\left|\Phi_{u} \cup B_{u}\right|=\varphi_{u}+b_{u}$, which by (3) gives $|M| \geq 2\left(\varphi_{u}+b_{u}\right) \geq \varphi_{u}+b_{u}+1$.

Finally, if $|\Upsilon(u)|=1$, i.e. $\ddot{u}=\dot{u}$, then $\left|\Phi_{u} \cup B_{u}\right|=\left|B_{u}\right|+|\{u\}|=b_{u}+1=\varphi_{u}$ and again by (3)

$$
|M| \geq 2\left|\Phi_{u} \cup B_{u}\right| \geq 2 \varphi_{u} \geq \varphi_{u}+b_{u}+1 .
$$

Lemma 3. Let $C$ be a longest cycle in a graph $G$ and $L$ a longest path in $G \backslash C$ with a $U_{0}$ minimal $L_{C}$-spreading $\Upsilon$. Then for each $u \in \bar{U}_{0},|L| \geq \varphi_{u}+b_{u}$.

Proof. Put $L=u_{1} \ldots u_{m}$. Let $\Lambda_{u}, \Lambda_{u}(x, y)$ and $\omega_{i}$ be as defined in proof of Lemma 2. Let $\xi_{1}, \ldots, \xi_{f}$ be the elements of $\Lambda_{u}$ occurring on $\vec{L}$ in a consecutive order. Set

$$
\vec{M}^{\prime}=u_{1} \vec{L} \xi_{1}, \quad \vec{M}^{\prime \prime}=\xi_{f} \vec{L} u_{m}, \quad \vec{M}_{i}=\xi_{i} \vec{L} \xi_{i+1} \quad(i=1, \ldots, f-1)
$$

Let $G^{\prime}$ be the graph obtained from $G$ by adding an extra edge $u_{m} u_{1}$. Set $\vec{M}=u_{1} \ldots u_{m} u_{1}$ and $M_{f}=\xi_{f} \vec{M} \xi_{1}$. Let $\Lambda_{u}^{\prime}\left(\xi_{f}, \xi_{1}\right)$ and $\Lambda_{u}^{\prime \prime}\left(\xi_{f}, \xi_{1}\right)$ be the paths obtained from $\Lambda_{u}\left(\xi_{f}, \xi_{1}\right)$ by deleting the first and the last edges, respectively. Since $L$ is extreme, $\left|M_{i}\right| \geq\left|\Lambda_{u}\left(\xi_{i}, \xi_{i+1}\right)\right|(i=1, \ldots, f-1)$. As for $M_{f}$, observe that

$$
\begin{aligned}
& \left|M^{\prime}\right| \geq\left|\Lambda_{u}^{\prime}\left(\xi_{f}, \xi_{1}\right)\right|=\left|\Lambda_{u}\left(\xi_{f}, \xi_{1}\right)\right|-1, \\
& \left|M^{\prime \prime}\right| \geq\left|\Lambda_{u}^{\prime \prime}\left(\xi_{f}, \xi_{1}\right)\right|=\left|\Lambda_{u}\left(\xi_{f}, \xi_{1}\right)\right|-1,
\end{aligned}
$$

Implying that

$$
\left|M_{f}\right|=\left|M^{\prime}\right|+\left|M^{\prime \prime}\right|+1 \geq 2\left|\Lambda_{u}\left(\xi_{f}, \xi_{1}\right)\right|-1 \geq\left|\Lambda_{u}\left(\xi_{f}, \xi_{1}\right)\right|
$$

So, $\left|M_{i}\right| \geq\left|\Lambda_{u}\left(\xi_{i}, \xi_{i+1}\right)\right|$ for each $i \in\{1, \ldots, f\}$. Further, for each $u \in U_{1}$, we can argue exactly as in proof of Lemma 2 to get $|L|=|M|-1 \geq \varphi_{u}+b_{u}$. Now let $u \in U^{*}$. By the definition, $\Phi_{u} \subseteq V(\Upsilon(u))$ and therefore, $\quad|\Upsilon(u)| \geq\left|\Phi_{u}\right|=\varphi_{u}$. Since $L$ is extreme, $|L| \geq 2\left(\left|B_{u}\right|+|\{u\}|\right)-2=2 b_{u}$. Hence,

$$
|L| \geq|\Upsilon(u)|+\frac{1}{2}|\vec{L}| \geq \varphi_{u}+b_{u} .
$$

Proof of Theorem 21 (Zh.G. Nikoghosyan, 1998). Let $M$ be a longest path in $G \backslash C$ of length $\bar{p}$ with a $\left(U_{0}\right)$-minimal $M_{C}$-spreading $\Upsilon$. If $\bar{p}=-1$, i.e. $M$ is a Hamilton cycle, then $|C| \geq \delta+1=(\bar{p}+2)(\delta-\bar{p})$. Let $\bar{p} \geq 0$. We claim that 
(a1) if $u \in U_{0}$ and $v \in \bar{U}_{0}$ then $\Phi_{u} \cap V(\Upsilon(v)) \subseteq\{v, \dot{v}\}$,

(a2) if $u \in U_{0}$ then $\varphi_{u} \leq \bar{p}+b_{u}^{*}$,

(a3) if $v \in \bar{U}_{0}$ then $\varphi_{u} \leq \bar{p}-b_{u}$.

Let $u \in U_{0}$. If $v \in \bar{U}_{0}$ then to prove (a1) we can argue exactly as in proof of Claim 2 (see the proof of Lemma 2). The next claim follows immediately from (a1). To prove (a3), let $v \in \bar{U}_{0}$. Since $M$ is extreme, by Lemma $3, \bar{p} \geq \varphi_{u}+b_{u}$ for each $u \in \bar{U}_{0}$, and (a3) follows.

Observing that

$$
\sum_{u \in U_{0}} b_{u}^{*}=\sum_{u \in \bar{U}_{0}} b_{u}
$$

and using (a2) and (a3), we get

$$
\sum_{u \in V(M)} \varphi_{u} \leq \bar{p}(\bar{p}+1)+\sum_{u \in U_{0}} b_{u}^{*}-\sum_{u \in \bar{U}_{0}} b_{u}=\bar{p}(\bar{p}+1) .
$$

Since $\Upsilon$ is extreme, we have

$$
\psi_{u}=d(\ddot{u})-\varphi_{u} \geq \delta-\varphi_{u}
$$

for each $u \in V(M)$.

By summing, we get

$$
\sum_{u \in V(M)} \psi_{u}=(\bar{p}+1) \delta-\sum_{u \in V(M)} \varphi_{u} \geq(\bar{p}+1)(\delta-\bar{p}) .
$$

In particular,

$$
\max _{u} \psi_{u} \geq \delta-\bar{p}
$$

By Lemma 1,

$$
\begin{aligned}
& |C| \geq \sum_{u \in V(M)} \psi_{u}+\max _{u} \psi_{u} \\
& \geq(\bar{p}+1)(\delta-\bar{p})+\delta-\bar{p}=(\bar{p}+2)(\delta-\bar{p}) .
\end{aligned}
$$

\section{Conclusions}

Graph invariants provide a powerful and maybe the single analytical tool for investigation of abstract structures of graphs. They, combined in convenient algebraic relations, carry global and general information about a graph and its particular substructures such as cycle 
structures, factors, matchings, colorings, coverings, and so on. The discovery of these relations is the primary problem of graph theory.

We focus on large cycle substructures, perhaps the most important cycle structures in graphs: Hamilton, longest and dominating cycles and some generalized cycles including Hamilton and dominating cycles as special cases.

In the literature, eight basic (initial) invariants of a graph $G$ are known having significant impact on large cycle structures, namely order $n$, size $q$, minimum degree $\delta$, connectivity $\kappa$, independence number $\alpha$, toughness $\tau$ and the lengths of a longest path and a longest cycle in $G \backslash C$ for a given longest cycle $C$ in $G$.

We have collected 37 pure algebraic relations between $n, q, \delta, \kappa, \alpha, \tau, \bar{p}$ and $\bar{c}$ ensuring the existence of a certain type of large cycles. The majority of these results are sharp in all respects.

Focusing only on basic graph invariants, as well as on pure algebraic relations between these parameters, in fact, we present the simplest kind of relations for large cycles having no forerunners in the area. Actually they form a source from which nearly all possible hamiltonian results (including well-known Ore's theorem, Pósa's theorem and many other generalizations) can be developed further by various additional new ideas, generalizations, extensions, restrictions and structural limitations.

\section{References}

Alon, N. (1986). The longest cycle of a graph with a large minimum degree, Journal of Graph Theory, Vol. 10, No. 1, pp. (123-127).

Bauer, D. \& Schmeichel, E. (1986). Long cycles in tough graphs, Technical Report 8612, Stevens Institute of Technology, Hoboken.

Bauer, D., Schmeichel, E. \& Veldman, H.J. (1988). A generalization of a Theorem of Bigalke and Jung, Ars Combinatoria, Vol. 26, pp. (53-58).

Bauer, D., Hakimi, S.L. \& Schmeichel, E. (1990a). Recognizing tough graphs is NP -hard, Discrete Applied Mathematics, Vol. 28, No. 3, pp. (191-195).

Bauer, D., Morgana, A., Schmeichel, E. \& Veldman, H.J. (1990b). Long cycles in graphs with large degree sums, Discrete Mathematics, Vol. 79, No. 1, pp. (59-70).

Bauer, D., Chen, G. \& Lasser, L. (1991a). A degree condition for Hamilton cycles in $t$-tough graphs with $t>1$, In: Advances in graph theory, V.R. Kulli, (Ed.), pp. (20-33), Vishwa International publications, Gulbarga, India.

Bauer, D. \& Schmeichel, E. (1991b). On a Theorem of Häggkvist and Nicoghossian, Graph Theory, Combinatorics, Algorithms and Applications, pp. (20-25).

Bermond, J.C. (1978). Hamiltonian graphs, In: Selected topics in graph theory, L. Beineke and R.J. Wilson, (Eds.), Academic press, London.

Bigalke, A. \& Jung, H.A. (1979). Über Hamiltonsche Kreise und unabhängige Ecken in Graphen, Monatshefte für Mathematik, Vol. 88, No. 3, pp. (195-210).

Bondy, J.A. \& Murty, U.S.R. (1976). Graph Theory with Applications, Macmillan, London, ISBN 0-444-19451-7. 
Bondy, J.A. (1981). Integrity in graph theory, In: The Theory and Application of Graphs, G. Chartrand, Y. Alavi, D.L. Goldsmith, L. Lesniak-Foster, D.R. Lick (Eds.), Wiley, New York, pp. (117-125). MR83e:05070.

Chvátal, V. \& Erdös, P. (1972). A note on hamiltonian circuits, Discrete Mathematics, Vol. 2, No. 2, pp. (111-113).

Chvátal, V. (1973). Tough graphs and Hamiltonian circuits, Discrete Mathematics, Vol. 5, No. 3 , pp. (215-228).

Dirac, G.A. (1952). Some theorems on abstract graphs, Proceedings of the London Mathematical Society, Vol. 2, No. 1, pp. (69-81).

Even, S. \& Tarjan, R.E. (1975). Network flow and testing graph connectivity, SIAM journal on computing, Vol. 4, No. 4, pp. (507-518).

Erdös, P. \& Gallai, T. (1959). On maximal paths and circuits of graphs, Acta Mathematica Hungarica, Vol. 10, No. 3-4, pp. (337-356).

Fraisse, P. (1986). $D_{\lambda}$-cycles and their applications for Hamiltonian graphs, Universite de Paris-sud, preprint.

Garey, M.R. \& Johnson, D.S. (1983). Computers and Intractability: A Guide to the Theory of NPCompletenes, Freeman, New York.

Gould, R.J. (1991). Updating the Hamiltonian Problem - A survey, Journal of Graph Theory, Vol. 15, No. 2, pp. (121-157).

Gould, R.J. (2003). Advances on the Hamiltonian Problem - A survey, Graphs and Combinatorics,Vol. 19, No. 1, pp. (7-52).

Häggkvist, R. \& Nicoghossian, G.G. (1981). A remark on hamiltonian cycles, Journal of Combinatorial Theory, Ser. B, Vol. 30, No. 1, pp. (118-120).

Jung, H.A. (1978). On maximal circuits in finite graphs, Annals of Discrete Mathematics, Vol. 3, pp. (129-144).

Jung, H.A. (1990). Long Cycles in Graphs with Moderate Connectivity, In: Topics in combinatorics and graph theory, R.Bodendieck and R.Henn (Eds), Phisika Verlag, Heidelberg, pp. (765-778).

Lu, M., Liu, H. \& Tian, F. (2005). Two sufficient conditions for dominating cycles, Journal of Graph Theory, Vol. 49, No. 2, pp. (135-150).

Mosesyan, C.M., Nikoghosyan, M.Zh. \& Nikoghosyan, Zh.G. (2009). Simple proofs of two Dirac-type theorems involving connectivity, In: arXiv:0906.3630v2 [math.CO], 27 Jul 2009, Available from: http://arxiv.org/abs/0906.3630.

Nash-Williams, C.St.J.A. (1971). Edge-disjoint hamiltonian cycles in graphs with vertices of large valency, In: Studies in Pure Mathematics, L. Mirsky, (Ed.), pp. (157-183), Academic Press, San Diego/London.

Nikoghosyan, M.Zh. \& Nikoghosyan, Zh.G. (2011). Large cycles in 4-connected graphs, Discrete Mathematics, Vol. 311, No. 4, pp. (302-306).

Nikoghosyan, Zh.G. (1981). On maximal cycle of a graph, DAN Arm. SSR, Vol. LXXII, No. 2, pp. (82-87) (in Russian).

Nikoghosyan, Zh.G. (1985a). A sufficient condition for a graph to be Hamiltonian, Matematicheskie voprosy kibernetiki $i$ vichislitelnoy tekhniki, Vol. 14, pp. (34-54) (in Russian). 
Nikoghosyan, Zh.G. (1985b). On maximal cycles in graphs, DAN Arm. SSR, Vol. LXXXI, No. 4, pp. (166-170) (in Russian).

Nikoghosyan, Zh.G. (1998). Path-Extensions and Long Cycles in Graphs, Mathematical Problems of Computer Science, Transactions of the Institute for Informatics and Automation Problems of the NAS (Republic of Armenia) and Yerevan State University, Vol. 19, pp. (25-31).

Nikoghosyan, Zh.G. (2000a). Cycle-Extensions and Long Cycles in Graphs, Mathematical Problems of Computer Science, Transactions of the Institute for Informatics and Automation Problems of the NAS (Republic of Armenia) and Yerevan State University, Vol. 21, pp. (121-128).

Nikoghosyan, Zh.G. (2000b). Cycle-Extensions and Long Cycles in $\kappa$-connected Graphs, Mathematical Problems of Computer Science, Transactions of the Institute for Informatics and Automation Problems of the NAS (Republic of Armenia) and Yerevan State University, Vol. 21, pp. (129-155).

Nikoghosyan, Zh.G. (2009a). Dirac-type generalizations concerning large cycles in graphs, Discrete Mathematics, Vol. 309, No. 8, pp. (1925-1930).

Nikoghosyan, Zh.G. (2009b). On the circumference, connectivity and dominating cycles, In: arXiv:0906.1857v1 [math.CO], 10 Jun 2009, Available from: http://arxiv.org/abs/0906.1857.

Nikoghosyan, Zh.G. (2011). A Size Bound for Hamilton Cycles, In: arXiv:1107.2201v1 [math.CO] 12 Jul 2011, Available from: http://arxiv.org/abs/1107.2201.

Voss H.-J. \& Zuluaga, C. (1977). Maximale gerade und ungerade Kreise in Graphen I, Wiss. Z. Tech. Hochschule, Ilmenau, Vol. 4, pp. (57-70).

Yamashita, T. (2009). A degree sum condition with connectivity for relative length of longest paths and cycles, Discrete Mathematics, Vol. 309, No. 23-24, pp. (6503-6507). 


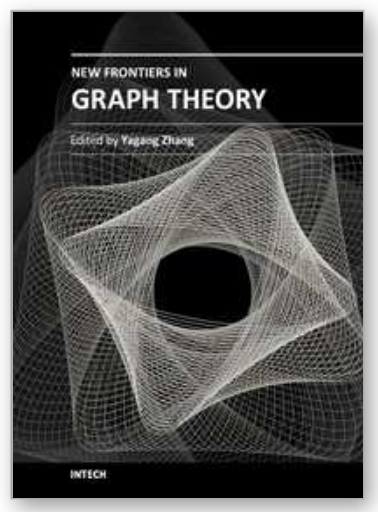

\author{
New Frontiers in Graph Theory \\ Edited by Dr. Yagang Zhang
}

ISBN 978-953-51-0115-4

Hard cover, 526 pages

Publisher InTech

Published online 02, March, 2012

Published in print edition March, 2012

Nowadays, graph theory is an important analysis tool in mathematics and computer science. Because of the inherent simplicity of graph theory, it can be used to model many different physical and abstract systems such as transportation and communication networks, models for business administration, political science, and psychology and so on. The purpose of this book is not only to present the latest state and development tendencies of graph theory, but to bring the reader far enough along the way to enable him to embark on the research problems of his own. Taking into account the large amount of knowledge about graph theory and practice presented in the book, it has two major parts: theoretical researches and applications. The book is also intended for both graduate and postgraduate students in fields such as mathematics, computer science, system sciences, biology, engineering, cybernetics, and social sciences, and as a reference for software professionals and practitioners.

\title{
How to reference
}

In order to correctly reference this scholarly work, feel free to copy and paste the following:

Zh.G. Nikoghosyan (2012). Pure Links Between Graph Invariants and Large Cycle Structures, New Frontiers in Graph Theory, Dr. Yagang Zhang (Ed.), ISBN: 978-953-51-0115-4, InTech, Available from:

http://www.intechopen.com/books/new-frontiers-in-graph-theory/pure-links-between-graph-invariants-andlarge-cycle-structures-

\section{INTECH}

open science | open minds

\section{InTech Europe}

University Campus STeP Ri

Slavka Krautzeka 83/A

51000 Rijeka, Croatia

Phone: +385 (51) 770447

Fax: +385 (51) 686166

www.intechopen.com

\section{InTech China}

Unit 405, Office Block, Hotel Equatorial Shanghai

No.65, Yan An Road (West), Shanghai, 200040, China

中国上海市延安西路65号上海国际贵都大饭店办公楼 405 单元

Phone: +86-21-62489820

Fax: $+86-21-62489821$ 
(C) 2012 The Author(s). Licensee IntechOpen. This is an open access article distributed under the terms of the Creative Commons Attribution 3.0 License, which permits unrestricted use, distribution, and reproduction in any medium, provided the original work is properly cited. 